\title{
Improvising Users Transaction Validation using Multi- Decision Tree Conditional of Terms in Block-Chain Verification
}

\author{
Yasser Al Sultan \\ Dept. of IS \\ King Abdul Aziz University \\ Jeddah, Saudi Arabia
}

\author{
Bassam A. Zafar \\ Dept. of CIT \\ King Abdul Aziz University \\ Jeddah, Saudi Arabia
}

\begin{abstract}
User's transactional validation in block chain at this time encounters a significant businesses fraud in block chain Validation. Research indicate that block chain algorithms having essential persistence had addressed security concepts influenced to carry out safety of user's contexts. Regulation how decentralized solutions can reduce the extent of transactions -fraud. In this paper a spot on a review of one other than bitcoin mining vulnerability various swapping's with larger blocks that compromise the security of block reliability causing a bit inconsistency to guarantee security leading to a replicas attempts. A Multi-Decision Tree algorithm set with a Conditional of Terms (CART) to Validates and updates transactions method supported with a multiple conditional terms of verifications is proposed to check the validity of the transactions pulled into a block, afterwards design interface to implement outcomes of data accuracy and fraud detection from the compared tested values. Result, and discussion make evident that validity of every transactions in a block either fulfilled, or violated in the terms of the conditions shown in flow-chart exhibited into both tables, and figure diagrams. Result point out simulation exchanges they are none redundant and validity between each user merely take place once.
\end{abstract}

\section{Keywords}

Block chain validation and verification, Decision Tree classifier, python

\section{INTRODUCTION}

Block chain, defined by Hilleman, described primary features in academia and industry, both cryptocurrencies is currently a new buzzword. Bitcoin is one of the most successful cryptocurrencies since it was the initial production of the block-chain system, and it enjoys tremendous victory with their capital market getting ten billion dollars in it architecture. [1]. cryptocurrency miners rapid interests had grown each year, and the objective is to take account of the features that supplementary towards less cost of transactions, and tremendous reliance, and assurance on user privacy .Producing a mechanism of this medium as it demanded to be electronic payments [2], Efficiency in relation to authentication can be improved using extra layer choices, and the cost can also be saved with all these attributes of the block chain [3].Even though the technology of block chain is having an excellent perspective on the development of Internet systems in the future, it is still facing numerous challenges. Data duplicating in transaction's-venerability is one of the significant concerns of block chain technology [4]. In formation of cryptocurrencies environment to enable electronic-money, and since this vital technology is allowing the payments to be made without any Intermediary. [5]. Thus, stimulated by decision Tree algorithm. It segments centered decision-making logic that can grip great

Dimensional data by means of decent accuracy.[6]. Nevertheless, based on a scientific research of decision tree several issues occurred in single classifiers by using that opportunely, a fascinating solution is approached in commended to apply multiple internal dictions nodes coded by python on this process [7]. Furthermore analyst suggested a digital track inspector to detect e-cash fraud attempts, which is robust, and secure designed to a tool system which assists in the review of digital confirmation that uses automation and artificial intelligence solution to treat with the cumulative complexity and volume of digital validation. [8], authors modeled multiple agent that concerning altered flowchart fraud cases that lead for earlier alert detection, using adaptive data-mining, and $\mathrm{AI}$ agents over time at different rates [9], targeting to minimize false alarm in fraud level Barred issues in business systems by applying HiddenMarkov method which allowed him elevate coverage collectively to less false alarm-ratio.[10], authors classified fraud using supervised-tactic is authorized by via examining the former records with the ones in progress from top-tobottom using a decision-tree method throughout low, medium, or high spending's to catch a fraud-act.[11], Block chain recently grew remarkable courtesy in regard of business system to the attention of developer's python site [12], Designed a health-care system that validate the metadata sent to each relay server via network using hash values to ensure the data reliability though their clients mobile devices , to avoid imitation by way of embedding a client hashing done into block chain techniques, methodology worked to register within that network.[13], In order to certify data integrity for user privacy, a design, and develop iterative cycle executed to prevent fraudulent manipulation of carriage mileage information, in addition a pre-post evaluation with multi use case conditions used. [14], The patent underlined importance procedure where a device identifiers linked by computingdevices offer an extra layer of security causing, the individual approved owns the proper gift-card number to transfer the gift card, such because the data is absolute and cannot be altered, therefore accumulate the security to any purchase.[15], Authors stated Unsupervised methods overall, and clustering techniques specifically, prospective thrives to determine unexpected patterns to the path uncovering valued insights, the first approach is using set of rules for cluster scaling improvement such is normalization, secondly approaching precise straight utilization characteristics towards the block chain data.[16]. In this paper, the idea is a multiple Decision Tree verified beforehand then validated later. Originally every single block was hashed as one with set of contents. The 
python hash library used to hash the contents called the module matplotlib.

To expresses all distribution of each block a plot bar chart diagram projected in the sequence. The software analysis included python programming language with IDE of Jupiter notebook. [17].

\section{PROBLEM STATEMENT}

Cross-bitcoin activities lack of verification by far the most conspicuous problem in the credit information-system complexity commissioned with the potential to considerably increase operational costs and data risk integrity. First and foremost, businesses has frequent intermediaries, a great risk of illegal transactions, high-costs, low-efficiency, may fear reduce the competence of supply-chain finance and increase manual operational risks. That is, once a piece of transaction pass in the system, it might be adjusted. Therefore, there must be firmer information access methods needed to be established, and the operations of each transaction on each block must be approved to confirm that fraud actions never occur.

\section{THE PROPOSED DECISION TREE}

Figure 1 CART flowchart consisted of 2 multi-layer verification decisions and 3 blocks that the validation had to check with a 3 conditions scale explained well along in testing, analysis, and simulation result and discussion. The objective was to conduct the validation each time the block is added to the block chain. A block is hashed with set of contents signed with a hash value. Any block doesn't meet the terms of second the decision layer is rejected, in order to avoid fraud $\mathrm{W}$ must equals to the TA, Resulting in return compliant to create the first block, tracking the operations afterward by revalidating the next block in chain to ensure security, and integrity of each transaction back to the first decision layer to confirm and update every one of those transactions into a new block. Finally displaying the variation operations changes to compare values.

\section{TESTING \& ANALYSIS}

\subsection{The Chart Diagram}

Figure 2 demonstrates how much coins transferred between separate users in the chain previously , and next each block transaction, blue indicates the first block for all users "genesis", the green block is the next block in the chain as you can see the sum of withdraw is as the magnitude of both deposits between all the users, finally the red shows a third block been created followed by the green the users still exchange coins as you can see in the chart both deposits is still enabled as long as the withdraw from the user applying two conditions subtracting isn't higher than what he owned in his actual amount, and neither equal to zero.

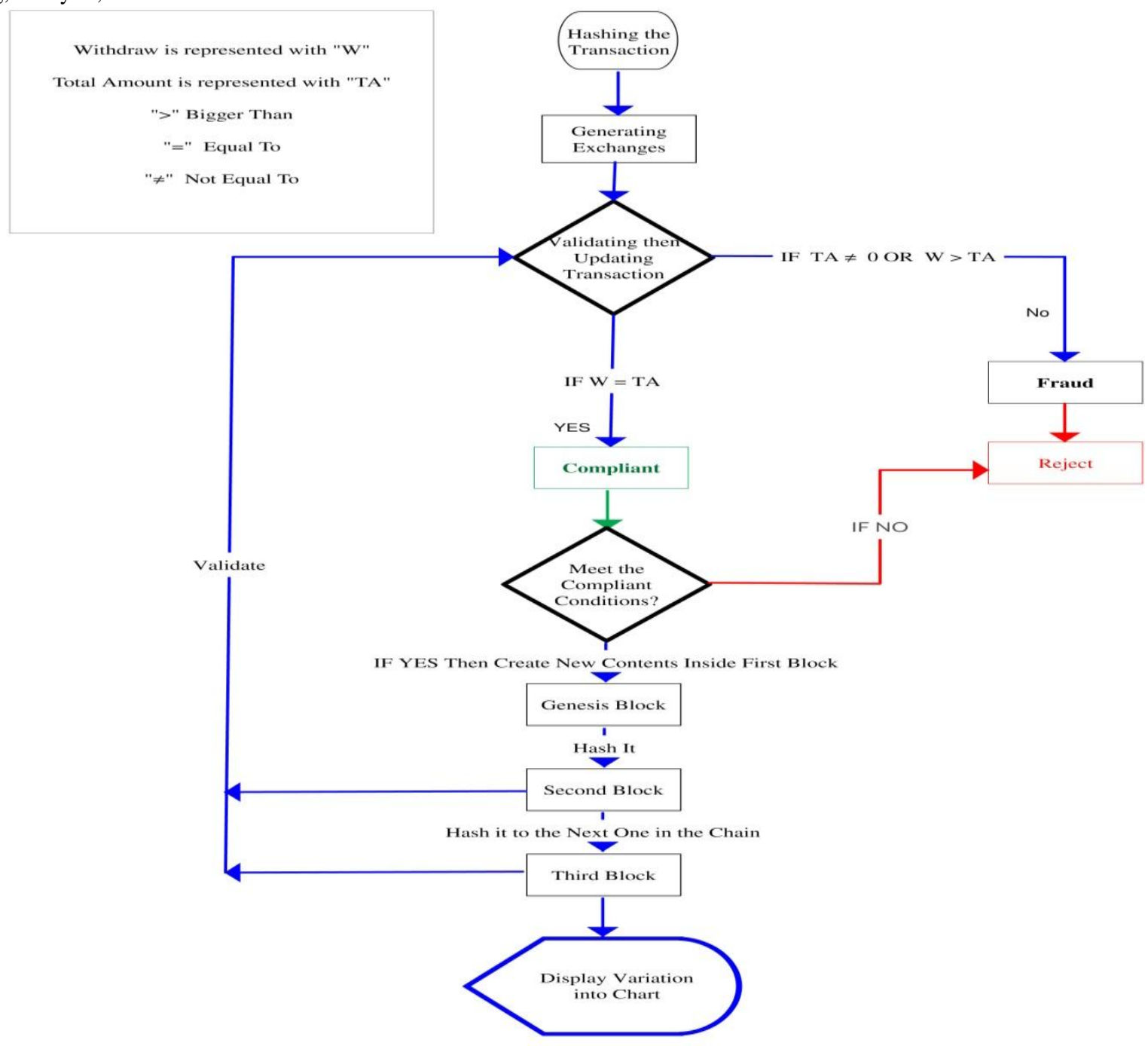

Figure 1 Flowchart for Multi- Decision Tree layer Verification 


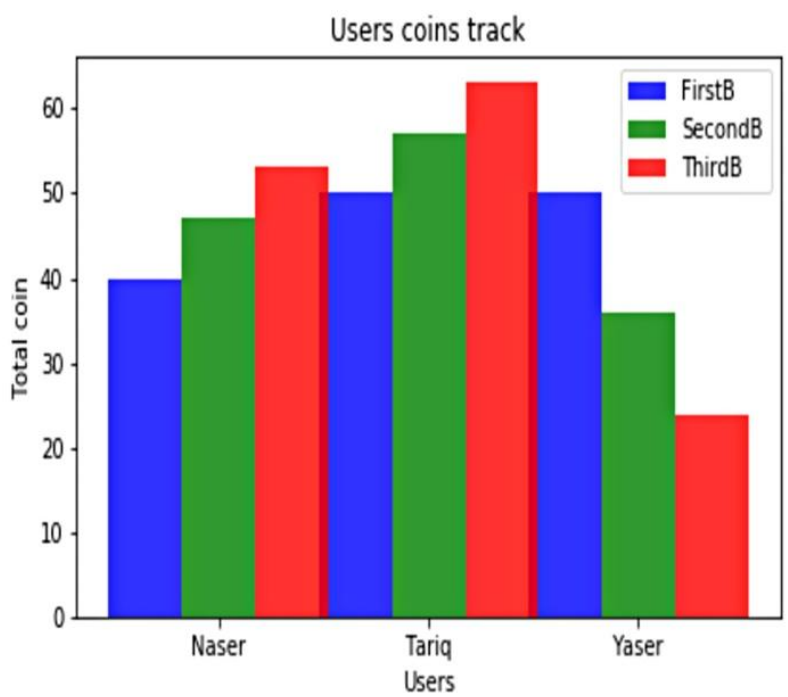

Figure 2 Block in the Chain Before, and After

\subsection{Interface Design}

Figure 3 Buttons in the interface are calculated for each entered inputs by using the math of:

(("Withdraws" + "Deposits") - "Total Amount") = "State".

The state is either true or false, by meaning that if both inputs checks to validate, or meet conditions, the function BTN Boolean code condition will check the values and compare them using this math:

$\mathrm{TA} \neq 0, \mathrm{~W}>\mathrm{TA}=\mathrm{FALSE}$

\section{$\mathrm{TA} \leq=$ TRUE}

Then saves the state of that output into an excel sheet format.

\subsection{Comparing the Accuracy of Deposits \& Withdraws}

Figure 4 Deploys data-set extracted from the predesign to measure the accuracy of each $\mathrm{D}+\mathrm{W}$ using formula:

(D - W)

W ---- X $100=$ percent accuracy.

As a result it contributes valuable insights, a differentiate that all withdraw-s values are correlated with $50 \%$ which indicate that the sums of both $\mathrm{D}$ equals the same scale of the $\mathrm{W}$ values, in the other hand False accuracy values are not correlated in a linear line giving above the percentage rate.

\subsection{Clustering Pair Values of Withdraw \& Total Amount for Fraud Detection}

Figure 5 verifies, and validates to display a wide variety of data to exhibit all transaction quantities in the clustered elements of W+TA, are compared by the balance of $\mathrm{W}$, and $\mathrm{TA}$, then evaluated every pair of values for fraud detection:

$\mathrm{W}>\mathrm{TA}=$ "2" FRAUD

$\mathrm{TA} \leq \mathrm{W}=$ "3" COMPLIENT.

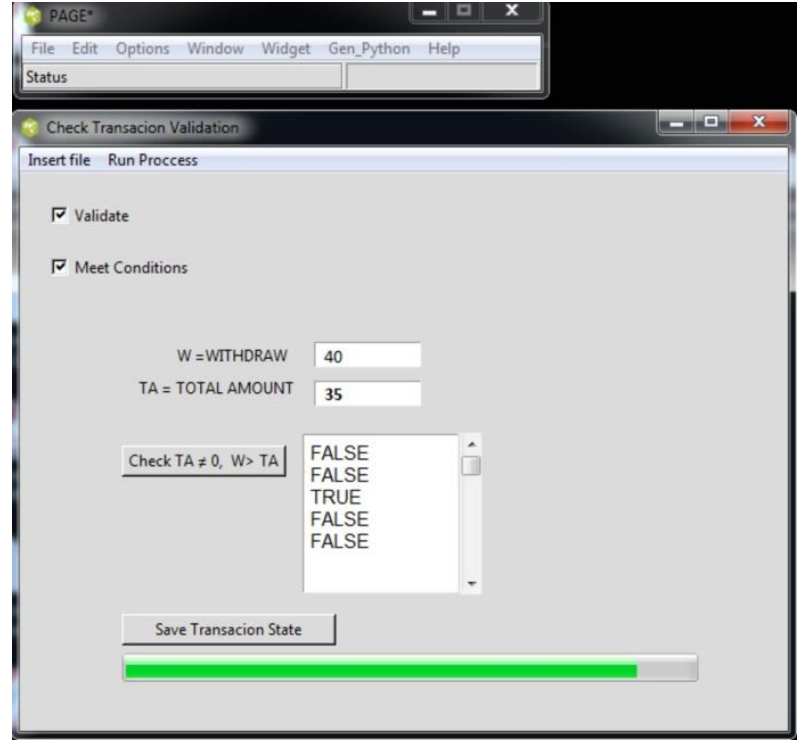

Figure 3 Interface design for Fraud Detection (Python Graphic Builder)

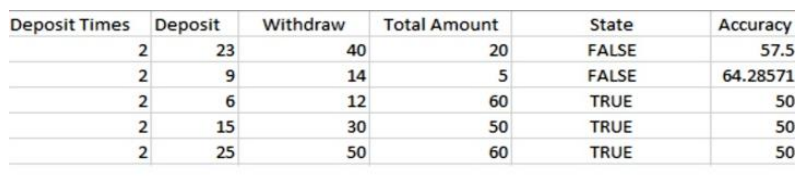

Comparing values of Withdraw and Total Amount for Fraud Detection

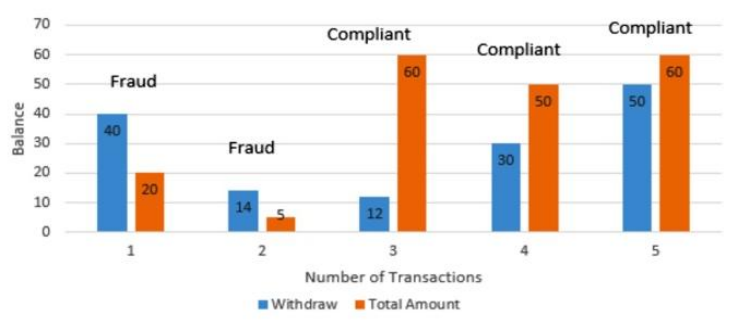

Figure 4 Calculating Accuracy of Transaction

\section{SIMULATION RESULTS \& DISCUSSIONS}

Based on the simulation result shown in Table 1, three users generated to exchange between Naser, Tariq and Yaser. The indication of withdrawals was per Subtract values, plus cashing in by addition ones.

Next in Table 2, and Table 3 constructing the trades always among coordinated exchangers, and making certain payment remains same amount similarly toward withdraws aren't generating or terminating currency, see Figure 1 the first transactions turned into a block, before all that there is the simulation condition that need to be defined to check the validity of those transactions pulled into that block.

Totality meant for operations essentially situated $=0$;

User accounts obligation devise plenty monetary each time cashing out;

The above conditions must be fulfilled, if either of them is violated it will reject the transaction;

Henceforth starting outlining genesis block. Since it never been associated just before former ones, In this case.

Create three user accounts (Naser, Tariq, Yaser );

Assign Tariq (50), and Yasser (50), then Naser at 
(40), its changeable For the first block;

Accumulate fixed transaction's, form a cyphered head tip, at that point added in next track;

- Miners check authenticity, then proceeds creating new upcoming blocks ;

Figure 2 finally shows that every chain block saved in a value for bar plotting chart;

\section{CONCLUSION}

In this work, Decision tree algorithm (CART) is recommended to resolve multiple validations for fraud selection problem. All users exchanged different transactions, those transactions can be subtracted from the total amount of each added block transaction, to ensure the integrity of the those transactions it had to deliver firmer conditions which are applied to whenever it does not full filled ultimately be rejected, therefore ensures security, and eliminate frauds the accuracy correspondence backs anticipated model conception. Next after each block the validity of that block is checked to make sure each block is associated to its former block, finally both states tables and variation plot results indicate that each block chain have different transaction number of sets, which shows concluding withdraws, and deposits that is plotted clearly into the clustering values illustration as a concrete detection proof. Resulting in nothing redundant from the results in the next chain. And the validity in transferring from one user to another happens only once which ensures a multilayered of verifications secured with authority method.

Table 4 Result of the Transaction shown as example in the block-chain contents

\begin{tabular}{|c|c|c|}
\hline \multirow{4}{*}{\begin{tabular}{c} 
Genesis block \\
\cline { 2 - 3 }
\end{tabular}} & Users & Balance \\
\cline { 2 - 3 } & Naser & 40 \\
\cline { 2 - 3 } & Tariq & 50 \\
\cline { 2 - 3 } & Yaser & 50 \\
\cline { 2 - 3 } & \{'Naser': 40, 'Tariq': 50, 'Yaser': 50 \\
\hline
\end{tabular}

Table 5 Result of the Transaction shown as example in the next block-chain contents

\begin{tabular}{|c|c|c|c|}
\hline Block 2 & Money & From & To \\
\hline Withdrawals & $-14 \$$ & - & Yaser \\
\hline Deposits & $7 \$$ & Yaser & Naser \\
\hline Deposits & $7 \$$ & Yaser & Tariq \\
\hline $\begin{array}{c}\text { New } \\
\text { Transaction }\end{array}$ & $\{$ 'Naser': 7, 'Tariq': 7, 'Yaser': -14 \} \\
\hline Result State & \multicolumn{2}{|c|}{ 'Naser': 47, 'Tariq': 57, 'Yaser': 36\} } \\
\hline
\end{tabular}

Table 6 Result of the Transaction shown as example below in block-chain contents

\begin{tabular}{|c|c|c|c|}
\hline Block 3 & Money & From & To \\
\hline Withdrawals & $-12 \$$ & - & Yaser \\
\hline Deposits & $6 \$$ & Yaser & Naser \\
\hline Deposits & $6 \$$ & Yaser & Tariq \\
\hline $\begin{array}{c}\text { New } \\
\text { Transaction }\end{array}$ & \multicolumn{2}{|c|}{ 'Naser': 6, 'Tariq': 6, 'Yaser': -12\} } \\
\hline Result State & \multicolumn{2}{|c|}{ 'Naser': 53, 'Tariq': 63, 'Yaser': 24\} } \\
\hline
\end{tabular}

\section{REFERENCES}

[1] Hileman, G. (2016). State of blockchain q1 2016: Blockchain funding overtakes bitcoin. CoinDesk, New York, NY, May, 11.

[2] Akins, B. W., Chapman, J. L., \& Gordon, J. M. (2014). A whole new world: Income tax considerations of the Bitcoin economy. Pitt. Tax Rev., 12, 25.

[3] Zhang, Y., \& Wen, J. (2015, February). An IoT electric business model based on the protocol of bitcoin. In Intelligence in Next Generation Networks (ICIN), 2015 18th International Conference on (pp. 184-191). IEEE

[4] Eyal, I., \& Sirer, E. G. (2018). Majority is not enough: Bitcoin mining is vulnerable. Communications of the ACM, 61(7), 95-102.

[5] CARTArticlehttps://www.datacamp.com/community/tut orials/decision-tree-classification-python

[6] [6] Kiran, S. (2018). Decision Tree Analysis Tool with the Design Approach of Probability Density Function towards Uncertain Data Classification'. International

[7] Journal of Scientific Research in Science and Technology (IJSRST), Print ISSN, 2395-6011.

[8] Henseler, H., Hofste, J., \& van Keulen, M. (2013, August). Digital-forensics based pattern recognition for

[9] Discovering identities in electronic evidence. In 2013 European Intelligence and Security Informatics Conference (pp. 112-116). IEEE.

[10] B.C., Amanze \& H.C, Inyiama \& Onyesolu, Moses. (2018). On the Development of Credit Card Fraud Detection System using Multi-Agents. International Journal of Computer Sciences and Engineering. 6. 13331343. 10.26438/ijcse/v6i6.13331343.

[11] Dhok, S.S. (2012). Credit Card Fraud Detection Using Hidden Markov Model.

[12] Singh, P., \& Singh, M. (2015). Fraud detection by monitoring customer behavior and activities. International Journal of Computer Applications, 111(11).

[13] Motohashi, T., Hirano, T., Okumura, K., Kashiyama, M., Ichikawa, D., \& Ueno, T. (2019). Secure and Scalable mHealth Data Management Using Blockchain Combined with Client Hashchain: System Design and Validation. Journal of medical Internet research, 21(5), e13385.

[14] Chanson, M., Bogner, A., Bilgeri, D., Fleisch, E., \& Wortmann, F. (2019). Privacy-Preserving Data Certification in the Internet of Things: Leveraging Blockchain Technology to Protect Sensor Data. Journal of the Association for Information Systems.

[15] Arora, A., Sengupta, S., Maheshwari, N., Kumawat, J. S., \& Bishnoi, V. (2019). U.S. Patent Application No. 15/794,322.

[16] Griffin, P. R., Megargel, A., \& Shankararaman, V. R. (2019). A Decision Framework for Decentralized Control of Distributed Processes: Is Blockchain the Only Solution? In Architectures and Frameworks for Developing and Applying Blockchain Technology (pp. 127). IGI Global.

[17] PYTHONLibraryhttps://github.com/blockcypher/blockcy pher-python. 